\title{
Aprendizaje-Servicio universitario en contextos de actividad física, educación física y deporte: una revisión sistemática
}

\author{
Laura Cañadas ${ }^{1}$ \\ ORCID: 0000-0003-4179-9018
}

\section{Resumen}

El Aprendizaje-Servicio ha mostrado ser una metodología muy útil en los Grados Universitarios donde el alumnado debe desarrollar competencias para intervenir en contextos donde desarrollará su actividad profesional, como aquellos del campo de la actividad física. El objetivo de este artículo es revisar sistemáticamente la evidencia existente sobre los efectos que la implementación de propuestas de Aprendizaje-Servicio en contextos de Actividad Física, Educación Física y Deporte tiene en los diferentes agentes implicados. Se llevó a cabo una revisión sistemática en tres bases de datos: Web of Science, ERIC and SportDiscus. Se encontraron 284 artículos, pero después del proceso de exclusión quedaron un total de 25. Los estudios muestran que la implementación de propuestas de Aprendizaje-Servicio tiene un impacto positivo en las competencias curriculares, sociales, culturales y cívicas, en el desarrollo e identidad profesional y las emociones. Solo 4 investigaciones han estudiado el impacto en los receptores del servicio, mostrando diferentes resultados en función del programa implementado. Solo un estudio evalúa el programa, mostrando buenos resultados. El Aprendizaje-Servicio en estos contextos ha mostrado numerosos beneficios para los estudiantes en el nivel académico, social y laboral; y en los receptores beneficiándose de la práctica de actividad física, y mejorando su salud y funciones motoras.

\section{Palabras clave}

Aprendizaje-Servicio - Universidad - Actividad física - Educación física - Deporte.

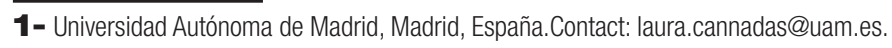




\section{University Service-Learning in physical activity, physical education, and sport settings: a systematic review}

\section{Abstract}

Service-Learning seems to be a very useful methodology in University Grades where students must develop competences to intervene in different contexts where they will be able to perform their professional activity such as those related with physical activity. The aim of this research is to conduct a systematic review of the evidence about the effects that university Service-Learning programs in Physical Activity, Physical Education and Sport settings have on the different agents involved. A systematic review was carried out by searching three databases: Web of Science, ERIC, and SportDiscus. We found 284 articles, but after the exclusion process, we ended up with 25. Studies have shown that implementing Service-Learning proposals have a positive impact in curricular, social, cultural, and civic competences, professional development, personal identity, and feelings. Only 4 researches address the impact on the recipients, showing different results depending on the program implemented. Just one study assesses the own program showing good results. University Service-Learning in these contexts has shown numerous benefits for students on the academic, social, and work levels; and for the recipients, it helps them make the most of the benefits of practicing physical activity, improving their health and motor functions.

\section{Keywords}

Service-learning - University - Physical activity - Physical education - Sport.

\section{Introducción}

La formación universitaria debe ser capaz de dar respuesta a las necesidades formativas de los futuros profesionales de la actividad física (AF), el deporte y docentes de educación física (EF). Esta formación debe dotarles de conocimientos concretos del campo de estudio, como hábitos saludables, condición física, expresión corporal, deportes, juegos, así como de competencias para intervenir en los diferentes contextos donde podrán desarrollar su labor profesional (SIEDENTOP, 2002). Al finalizar su formación deben ser capaces de programar, intervenir y evaluar propuestas específicas de actividad física y deporte. Además, esta formación debe contribuir a que sean capaces de adecuarse a diferentes contextos laborales, entre ellos, deportivos, educativos, recreativos y saludables, respondiendo a los objetivos específicos a desarrollar en cada uno de ellos. Esta formación también debe adaptarse a los actuales retos y necesidades sociales, contribuyendo a desarrollar prácticas de actividad física de calidad con diferentes colectivos y poblaciones: 
niños, adolescentes, adultos, personas con discapacidad, personas en riesgo de exclusión social, colectivos de mujeres, adultos mayores, etc.

Para ello desde la formación inicial se busca utilizar estrategias metodológicas que cubran al máximo posible esta diversidad de necesidades formativas, así como conseguir un aprendizaje más profundo y duradero. Entre estas metodologías el Aprendizaje-Servicio (ApS) ha tenido un fuerte impulso en los últimos años (LOUGH, 2018). Los fundamentos teóricos y prácticos del ApS proceden del aprendizaje experiencial y el constructivismo enmarcando el aprendizaje como oportunidades para que el alumnado aplique el conocimiento que han aprendido en el aula en contextos comunitarios contribuyendo tanto al compromiso comunitario como al aprendizaje (FURCO; NORVELL, 2019). El ApS en el contexto universitario se define como "la integración de aprendizajes académicos, actividades de servicio relevantes en la comunidad y reflexión crítica, que se asocian de forma recíproca y que involucra a los estudiantes, profesores y miembros de la comunidad para lograr objetivos de aprendizaje académico, cívico y personal, así como para lograr fines sociales" (BRINGLE; CLAYTON, 2012, p. 105).

En el contexto universitario su utilización está cada vez más extendida, ya que ha mostrado tener beneficios para el alumnado. De forma general esto se pueden agrupar en seis grandes categorías: (a) desarrollo académico (aprendizajes propios del área de estudio, calificaciones, habilidades cognitivas); (b) competencias sociales (habilidades interpersonales, relaciones sociales, capacidad para llegar a acuerdos con el resto de agentes, liderazgo); (c) competencias culturales y cívicas (comprendiendo la diversidad de etnias y poblaciones, transformación de las concepciones y creencias, la importancia de la responsabilidad social); (d) desarrollo personal (autoestima, auto-reconocimiento, motivación, empoderamiento, autoeficacia); (e) perspectiva profesional (confirmando la elección realizada o abriendo nuevas vías profesionales); y (f) consideraciones éticas (GARDNER; EMORY, 2018; HÉBERT; HAUF, 2015; WALKER, 2020). La otra parte que caracteriza al ApS es el servicio que proporciona a la comunidad en contextos desfavorecidos o de vulnerabilidad. Los proyectos de ApS deben ser diseñados partiendo de una necesidad concreta detectada en la comunidad. Estos deben ayudar a dar respuesta a esa necesidad que de otra forma no podría ser cubierta, contribuyendo al cambio local y al desarrollo. Por tanto, deben estar orientados a dar respuesta a los objetivos de la organización. En este sentido, tras llevar a cabo los proyectos, debe evaluarse tanto el cumplimiento de estos objetivos como el impacto que el programa ha tenido sobre los receptores (TRYON; STOECKER, 2008). Respecto a las organizaciones, la investigación ha mostrado los beneficios que produce en lo referente al intercambio cultural, las ventajas económicas y la transferencia de conocimiento y productividad (HARRINGTON, 2014). En los receptores directos, los cambios y mejoras son tan diversos como el servicio recibido.

Vista la importancia de esta metodología, en este artículo buscamos revisar sistemáticamente la evidencia existente sobre los efectos que la implementación de propuestas de Aprendizaje-Servicio en contextos de Actividad Física, Educación Física y Deporte tiene en los diferentes agentes implicados. 


\section{Métodos}

\section{Selección de la literatura}

Durante Noviembre de 2018 se realizó una revisión de la literatura en 3 bases de datos diferentes (WOS, Sportdiscus y ERIC) desde 2008 a 2018. Estas bases de datos se seleccionaron por ser repositorios de investigación de calidad. Además, ERIC es una de las bases más importantes de investigación educative. Los principales términos de búsqueda empleados fueron "service-learning" AND ("physical activity" OR "physical education" OR "health education” OR "sports”). En la Tabla 1 se encuentra una relación del número de artículos encontrados en las búsquedas en las bases de datos. Para corroborar la fiabilidad de los resultados encontrados se realizó una primera búsqueda y pasados 15 días se volvió a realizar la búsqueda con los mismos términos, comprobando que se encontraban los mismos resultados.

Tabla 1- Términos usados en la búsqueda

\begin{tabular}{|c|c|c|}
\hline Base de datos & Términos de búsqueda & $\mathrm{N}$ \\
\hline \multirow{3}{*}{ Web of Science } & Service-Learning + physical activity & 44 \\
\hline & Service-Learning + phsycal education & 46 \\
\hline & Service-Learning + Sport & 20 \\
\hline \multirow{3}{*}{ ERIC+Sportdiscus } & Service-Learning + physical activity & 39 \\
\hline & Service-Learning + phsycal education & 85 \\
\hline & Service-Learning + Sport & 50 \\
\hline \multicolumn{2}{|r|}{ Total } & 284 \\
\hline
\end{tabular}

Fuente: Elaboración propia.

\section{Criterios de inclusión}

Se establecieron una serie de criterios para la inclusión de los artículos en esta revisión sistemática. Cada investigación debía cumplir los siguientes criterios:

a) Criterio de idioma: artículo publicado en inglés o español.

b) Criterio de intervención: llevaban a cabo propuestas de Aprendizaje-Servicio con alumnado Universitario a través de intervenciones de actividad física (el alumnado universitario diseñase e interviniese a través de la dirección de juegos, tareas o sesiones de actividad física). Excluían aquellas intervenciones relacionadas con la gestión de eventos deportivos por no llevar a cabo el alumnado intervenciones concretas de actividad física.

c) Criterio de participantes: Incluyera estudiantes universitarios de titulaciones relacionadas con las Ciencias de la Actividad Física y el Deporte (Kinesiology, Health 
Education, Ciencias del Deporte), Educación Física (Formación de Profesorado de Educación Primaria o Secundaria) o Educación y/o Trabajo Social.

d) Criterio de investigación: Que presentase un diseño de investigación de carácter transversal o longitudinal. Se excluían las descripciones de experiencias.

e) Criterio de resultado: describen al menos un resultado producido por la implementación de una propuesta de ApS.

Para la extracción de la información se empleó una tabla con los datos de las investigaciones. Toda la información se encuentra en la Tabla 2. Esta tabla incluye detalle de: autor, año, país, quiénes fueron los receptores del servicio, la duración del proyecto de ApS (semanas y horas con el objetivo de determinar la duración del proyecto), metodología empleada, instrumentos utilizados, a quiénes se recoge la información de la investigación (participantes), qué aspecto del ApS se investiga, incluyendo aprendizaje, servicio, aprendizaje+servicio y principales resultados encontrados. Estos elementos se seleccionaron por su importancia en los proyectos de ApS.

Los receptores del servicio muestran en qué población se han realizado las intervenciones en actividad física y deporte y que contextos no fueron cubiertas; la duración del proyecto de ApS está directamente relacionada con el aprendizaje de los estudiantes universitarios y los posibles resultados del servicio; la metodología junto con los instrumentos empleados indicarán cuáles fueron los más utilizados las investigaciones y qué innovaciones se podrían realizar en esta área; conocer de qué agentes se ha recogido la información de la investigación nos ayudará a saber si se ha tenido en cuenta la perspectiva de todos los implicados o si se ha limitado a información parcial; qué aspectos del ApS se han investigado nos ayudarán a saber si solo se valoró una parte del proyecto, o el proyecto en su totalidad (generalmente, el aprendizaje de los estudiantes universitarios); finalmente, los resultados indicarán las principales conclusiones del impacto del ApS en los diferentes aspectos investigados.

\section{Resultados}

\section{Resultados de búsqueda}

La Figura 1 presenta el proceso llevado a cabo para la selección de los artículos incluidos en la revisión sistemática. En la búsqueda inicial se encontraron un total de 284 resultados. Tras una primera revisión se eliminaron 110 investigaciones duplicadas. Posteriormente, se pasaron a analizar los 174 artículos restantes a partir de sus resúmenes y títulos. Tras este análisis se eliminaron 89 investigaciones que no se relacionaban con la temática a analizar. Los 85 artículos restantes se analizaron a texto completo eliminando 60 que no cumplían alguno de los criterios de inclusión. Finalmente, 25 artículos fueron incluidos en la revisión sistemática. La información concreta de cada estudio se encuentra en la Tabla 2. 
Gráfico 1- Proceso de selección de artículos

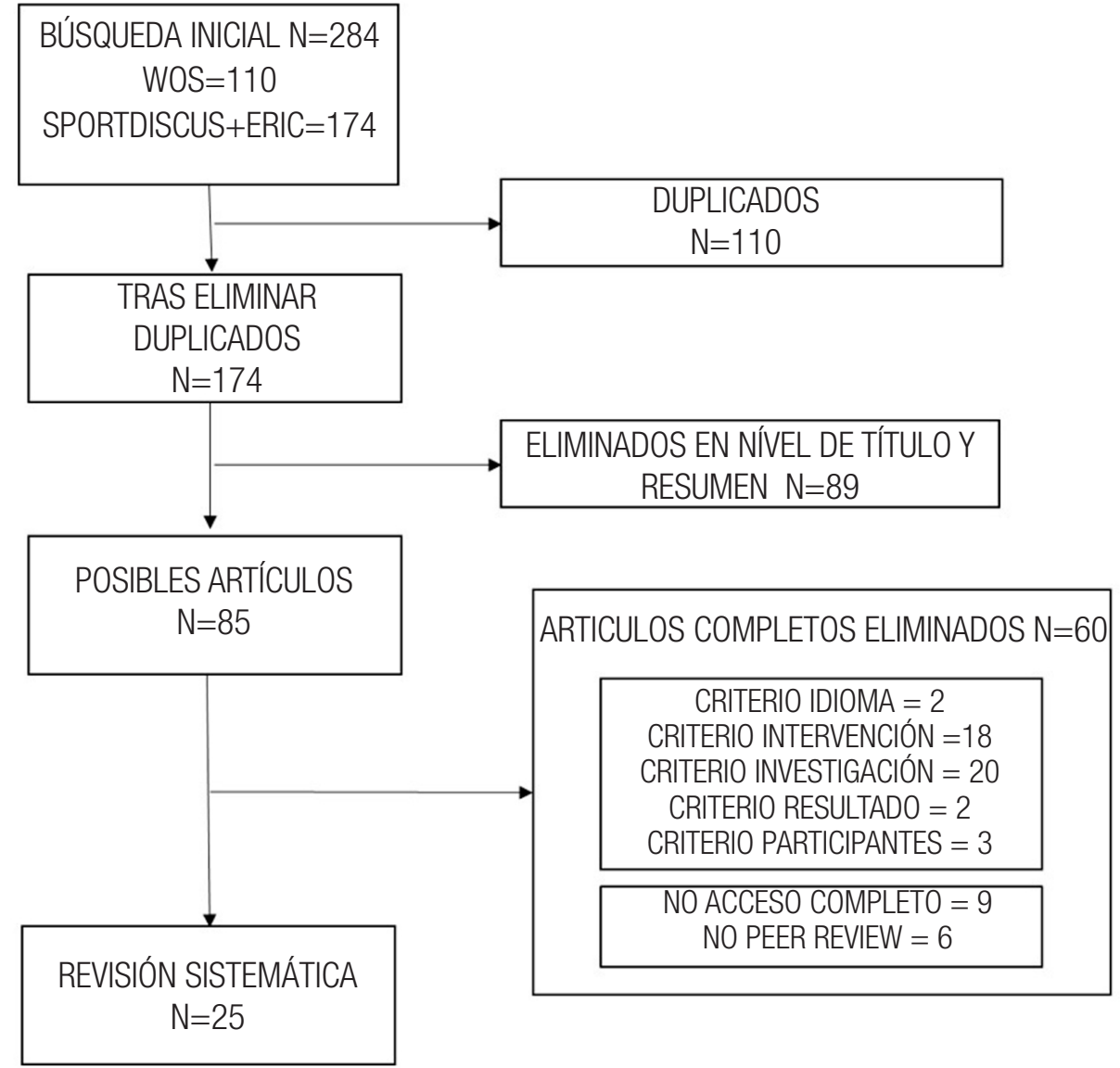

Fuente: Elaboración propia.

Table 2- Resumen de las investigaciones incluidas en esta investigación

\begin{tabular}{|c|c|c|c|c|c|c|c|c|}
\hline $\begin{array}{l}\mathscr{0} \\
\stackrel{0}{0} \\
\stackrel{+}{⿺}\end{array}$ & $\frac{\infty}{\mathbb{1 0}}$ & $\begin{array}{c}\text { Receptores } \\
\text { Servicio }\end{array}$ & Duración & 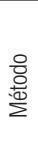 & 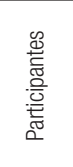 & 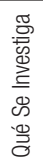 & Instrumentos & Resultados \\
\hline 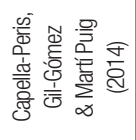 & 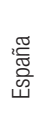 & $\begin{array}{l}\text { Niños/as con } \\
\text { diversidad } \\
\text { funcional }\end{array}$ & & 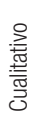 & 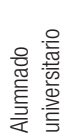 & 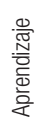 & Entrevistas abiertas & $\begin{array}{l}\text { Aprendizajes académicos } \\
\text { Desarrollo profesional } \\
\text { (resolución dudas } \\
\text { profesionales) }\end{array}$ \\
\hline 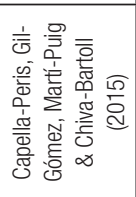 & 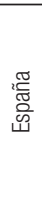 & $\begin{array}{l}\text { Niños/as con } \\
\text { diversidad } \\
\text { funcional }\end{array}$ & & 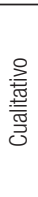 & 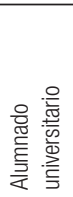 & 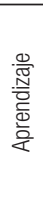 & Entrevistas abiertas & $\begin{array}{l}\text { Reafirmación vocación } \\
\text { Eliminación prejuicios y } \\
\text { comprensión de la diversidad } \\
\text { Desarrollo de valores de } \\
\text { carácter moral (cohesión social, } \\
\text { cooperación, empatía) }\end{array}$ \\
\hline
\end{tabular}


Aprendizaje-Servicio universitario en contextos de actividad física, educación física y deporte...

\begin{tabular}{|c|c|c|c|c|c|c|c|c|}
\hline 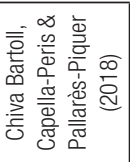 & 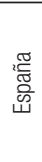 & $\begin{array}{l}\text { Niños/as con } \\
\text { alteraciones de la } \\
\text { motricidad }\end{array}$ & & 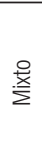 & 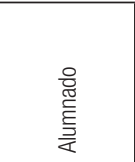 & 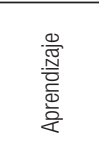 & $\begin{array}{l}\text { Cuestionario } \\
\text { Diarios de seguimiento } \\
\text { Entrevistas }\end{array}$ & $\begin{array}{l}\text { Mejora habilidad comunicativa } \\
\text { y en la implicación y } \\
\text { organización grupal }\end{array}$ \\
\hline 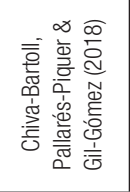 & 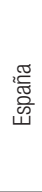 & $\begin{array}{l}\text { Niños/as con } \\
\text { diversidad } \\
\text { funcional }\end{array}$ & & $\stackrel{0}{i x}$ & 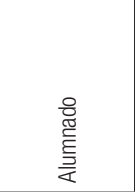 & 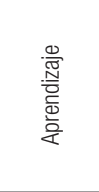 & $\begin{array}{l}\text { Cualitativo: Entrevistas y } \\
\text { diarios de seguimiento } \\
\text { Cuantitativo: Cuestionario } \\
\text { de Personalidad Eficaz } \\
\text { para universitarios }\end{array}$ & $\begin{array}{l}\text { Mejora planificación y toma de } \\
\text { decisiones. Unión entre teoría } \\
\text { y práctica } \\
\text { Identidad personal: mejora } \\
\text { autoestima y auto-concepto } \\
\text { Interacción social }\end{array}$ \\
\hline 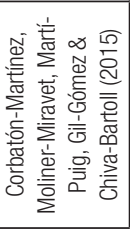 & 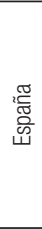 & $\begin{array}{l}\text { Niños y niñas con } \\
\text { TDAH }\end{array}$ & $\begin{array}{l}\text { Semanas: - } \\
\text { No de veces a la } \\
\text { semana: Una vez a la } \\
\text { semana } \\
\text { Horas/sesión: - } \\
\text { Horas totales: } 40-45 \\
\text { horas. }\end{array}$ & 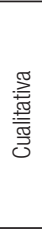 & 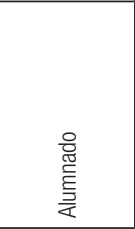 & 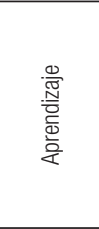 & $\begin{array}{l}\text { Reflexiones escritas } \\
\text { Grupo de discusión }\end{array}$ & $\begin{array}{l}\text { Competencias docentes } \\
\text { (autoeficacia, resolución de } \\
\text { problemas, gestión del aula) } \\
\text { Comprensión de las } \\
\text { necesidades del alumnado con } \\
\text { TDHA, implicación en la mejora } \\
\text { de la sociedad }\end{array}$ \\
\hline 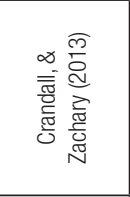 & 蛋 & $\begin{array}{l}\text { Colegios de } \\
\text { Primaria de zonas } \\
\text { altamente pobres }\end{array}$ & $\begin{array}{l}\text { Semanas: - } \\
\text { No de veces a la } \\
\text { semana: Una vez a } \\
\text { la semana } \\
\text { Horas/sesión: - } \\
\text { Horas totales: - } \\
\end{array}$ & 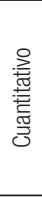 & 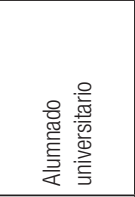 & 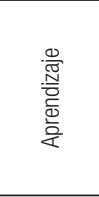 & $\begin{array}{l}\text { Openness to Diversity } \\
\text { and Challenge Scale }\end{array}$ & Apertura a la diversidad. \\
\hline 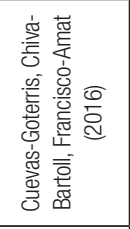 & 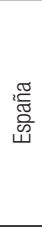 & $\begin{array}{l}\text { Niños con } \\
\text { diversidad } \\
\text { funcional }\end{array}$ & $\begin{array}{l}\text { Semanas: } 4 \\
\text { No de veces a la } \\
\text { semana: Una vez a la } \\
\text { semana } \\
\text { Horas/sesión: } 3 \text { horas } \\
\text { Horas totales: - }\end{array}$ & 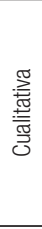 & 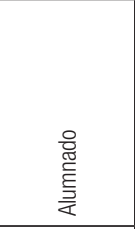 & 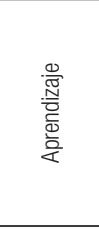 & $\begin{array}{l}\text { Entrevistas } \\
\text { semi-estructuradas } \\
\text { Grupo de discusión }\end{array}$ & $\begin{array}{l}\text { No mejora trabajo en grupo, } \\
\text { aunque contribuye a aprender } \\
\text { a relacionarse con otros } \\
\text { agentes } \\
\text { Mejora capacidad críticas y } \\
\text { reflexión sobre una realidad } \\
\text { que no conocían }\end{array}$ \\
\hline 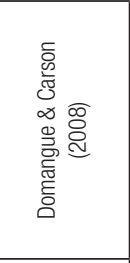 & 姿 & $\begin{array}{l}\text { Niños con un } \\
\text { nivel económico } \\
\text { bajo de una casa } \\
\text { comunitaria para } \\
\text { gente afectada } \\
\text { por el Katrina }\end{array}$ & $\begin{array}{l}\text { Semanas: - } \\
\text { No de veces a la } \\
\text { semana - } \\
\text { Horas/sesión: - } \\
\text { Horas totales: } 15 \\
\text { horas }\end{array}$ & 产 & 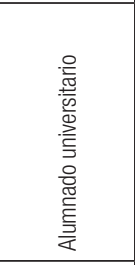 & 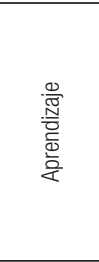 & $\begin{array}{l}\text { Cuestionario } \\
\text { (Multicultural Teaching } \\
\text { Competencies Scale) } \\
\text { Diarios de reflexión } \\
\text { Entrevista }\end{array}$ & $\begin{array}{l}\text { Mayores valores en los que } \\
\text { participaron en ApS que los } \\
\text { que no, y aumentó tras el } \\
\text { curso. Las reflexiones son } \\
\text { necesarias para establecer la } \\
\text { competencia cultural. Cambio } \\
\text { de perspectiva sobre los niños } \\
\text { con los que trabajaron. }\end{array}$ \\
\hline 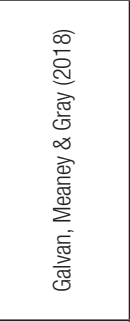 & 蛋 & $\begin{array}{l}\text { Niños con } \\
\text { sobrepeso y } \\
\text { poco interés en } \\
\text { el ejercicio, y } \\
\text { problemas de } \\
\text { comportamiento }\end{array}$ & $\begin{array}{l}\text { Semanas: } 10 \\
\text { № de veces a la } \\
\text { semana: - } \\
\text { Horas/sesión: - } \\
\text { Horas totales: } 20 \\
\text { horas. }\end{array}$ & $\frac{0}{x}$ & 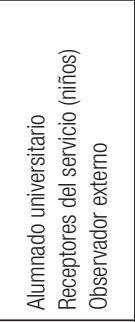 & 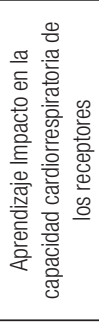 & $\begin{array}{l}\text { Test de una milla del } \\
\text { fitnessgram para } \\
\text { valorar la resistencia } \\
\text { cardiorrespiratoria. } \\
\text { Diarios reflexivos } \\
\text { Grupos de discusión } \\
\text { Notas de campo de los } \\
\text { investigadores. }\end{array}$ & $\begin{array}{l}\text { Servicio: Mejora de la } \\
\text { resistencia cardiorrespiratoria } \\
\text { Mejora aprendizaje del } \\
\text { contenido, mejora del } \\
\text { conocimiento pedagógico del } \\
\text { contenido } \\
\text { Desarrollo de la empatía por } \\
\text { los otros } \\
\text { Mejora las habilidades para } \\
\text { colaborar con otros } \\
\end{array}$ \\
\hline 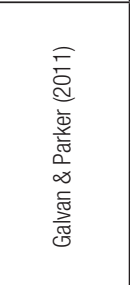 & 蛋 & $\begin{array}{l}\text { Organización sin } \\
\text { ánimo de lucro } \\
\text { para jóvenes } \\
\text { desatendidos }\end{array}$ & $\begin{array}{l}\text { Semanas: } 9 \\
\text { No de veces a la } \\
\text { semana: } 3 \text { días/ } \\
\text { semana } \\
\text { Horas totales: } 30-45^{\prime} \\
\text { Horas totales: - }\end{array}$ & 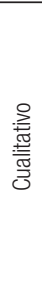 & 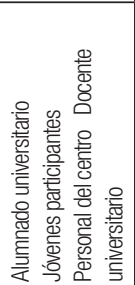 & 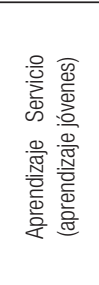 & $\begin{array}{l}\text { Entrevistas, notas de } \\
\text { campo y los diarios del } \\
\text { alumnado }\end{array}$ & $\begin{array}{l}\text { Aplicar el conocimiento } \\
\text { pedagógico del contenido } \\
\text { anteriormente adquirido en un } \\
\text { contexto real. Estrategias de } \\
\text { gestión del aula. Dan cuenta } \\
\text { que les falta conocimiento del } \\
\text { contenido } \\
\text { Se desarrolla solo de forma } \\
\text { superficial }\end{array}$ \\
\hline
\end{tabular}




\begin{tabular}{|c|c|c|c|c|c|c|c|c|}
\hline 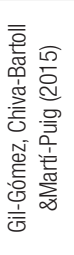 & 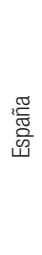 & $\begin{array}{l}\text { Niños con } \\
\text { necesidades } \\
\text { educativas } \\
\text { especiales }\end{array}$ & $\begin{array}{l}\text { Semanas: - } \\
\text { No de veces a la } \\
\text { semana: - } \\
\text { Horas/sesión: - } \\
\text { Horas totales: 40-45 } \\
\text { horas. }\end{array}$ & 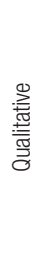 & 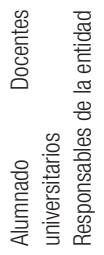 & 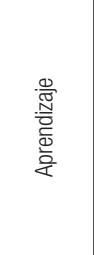 & $\begin{array}{l}\text { Diario reflexivo de los } \\
\text { estudiantes } \\
\text { Sesiones globales } \\
\text { de discusión con los } \\
\text { agentes implicados tras } \\
\text { cada intervención } \\
\text { Grupo de discusión de } \\
\text { expertos }\end{array}$ & $\begin{array}{l}\text { Mejora la competencia docente } \\
\text { Mejora la comprensión de la } \\
\text { discapacidad } \\
\text { No hay cambios remarcables }\end{array}$ \\
\hline 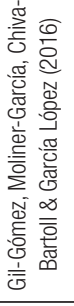 & 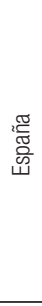 & $\begin{array}{l}\text { Niños y niñas } \\
\text { con problemas } \\
\text { de diversidad } \\
\text { funcional }\end{array}$ & $\begin{array}{l}\text { Semanas: } 11 \\
\text { No de veces a la } \\
\text { semana: - } \\
\text { Horas/sesión: - } \\
\text { Horas totales: } 136 \\
\text { horas. }\end{array}$ & $\frac{\sqrt[0]{x}}{2}$ & 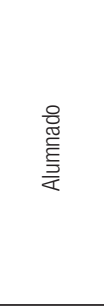 & 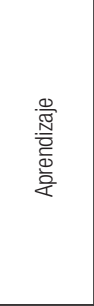 & $\begin{array}{l}\text { Cuestionario Evaluación de } \\
\text { la competencia Ciudadana } \\
\text { Prosocial e Inclusiva } \\
\text { Documento de Servicio } \\
\text { de la Asignatura } \\
\text { Diario de Seguimiento } \\
\text { del Servicio }\end{array}$ & $\begin{array}{l}\text { Mejora sensibilidad y } \\
\text { responsabilidad social }\end{array}$ \\
\hline 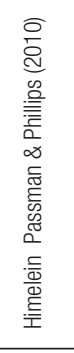 & 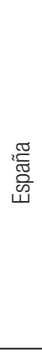 & $\begin{array}{l}\text { Familias } \\
\text { completas para } \\
\text { prevención } \\
\text { de obesidad } \\
\text { en contextos } \\
\text { desfavorecidos }\end{array}$ & $\begin{array}{l}\text { Semanas: - } \\
\text { No de veces a la } \\
\text { semana: - } \\
\text { Horas/sesión: - } \\
\text { Horas totales: } 14 \\
\text { horas }\end{array}$ & $\frac{0}{x}$ & 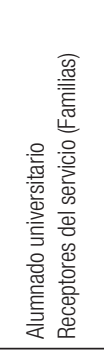 & 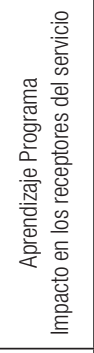 & $\begin{array}{l}\text { Cuestionario } \\
\text { Grupo de discusión } \\
\text { Entrevistas }\end{array}$ & $\begin{array}{l}\text { Aplicar el aprendizaje } \\
\text { académico a un problema } \\
\text { del mundo real. Mejora la } \\
\text { comprensión del contenido } \\
\text { del curso } \\
\text { Seguridad en sí mismo, } \\
\text { empatía, autoconciencia } \\
\text { Servicio: Se percibe un impacto } \\
\text { en el estilo de vida de las familias, } \\
\text { aumentando la AFy mejorando } \\
\text { los hábitos alimenticios } \\
\end{array}$ \\
\hline 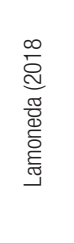 & 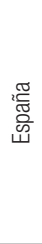 & $\begin{array}{l}\text { Alumnado } \\
\text { de centros } \\
\text { de Primaria } \\
\text { y Secundaria } \\
\text { (dinamización de } \\
\text { recreos) }\end{array}$ & 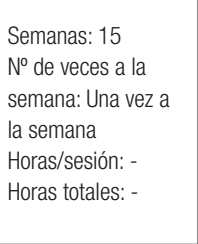 & 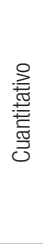 & 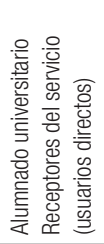 & 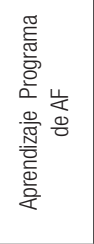 & $\begin{array}{l}\text { Cuestionario para } \\
\text { evaluar la calidad en } \\
\text { programas de Actividad } \\
\text { Física (Hernández, 2001). } \\
\text { Cuestionario de } \\
\text { competencias genéricas } \\
\text { creado ad hoc }\end{array}$ & $\begin{array}{l}\text { Programa: Se valoró } \\
\text { positivamente el programa, así } \\
\text { como la formación del monitor. } \\
\text { Planteaban aspectos a mejorar } \\
\text { Aprendizaje: Se valora } \\
\text { positivamente la adquisición de } \\
\text { competencias genéricas }\end{array}$ \\
\hline 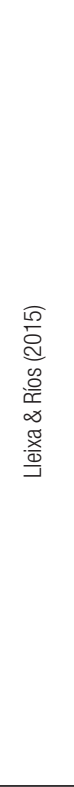 & 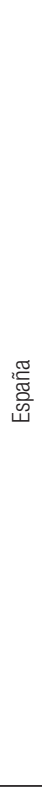 & $\begin{array}{l}\text { Presos de } \\
\text { la unidad } \\
\text { psiquiátrica }\end{array}$ & $\begin{array}{l}\text { Semanas: - } \\
\text { No de veces a la } \\
\text { semana: Una vez a } \\
\text { la semana } \\
\text { Horas/sesión: } 5 \\
\text { horas } \\
\text { Horas totales: - }\end{array}$ & 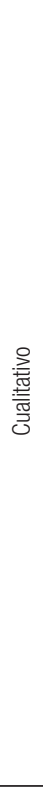 & 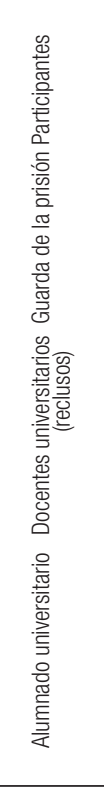 & 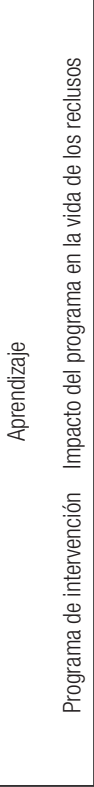 & $\begin{array}{l}\text { Entrevista semi- } \\
\text { estructurada con el } \\
\text { guarda de la prisión } \\
\text { Los diarios de } \\
\text { campo del alumnado } \\
2 \text { grupos de discusión: } \\
\text { uno con miembros del } \\
\text { grupo de trabajo y otro } \\
\text { con presos }\end{array}$ & $\begin{array}{l}\text { Aprendizaje } \\
\text { Ayuda a reducir el estigma } \\
\text { sobre la enfermedad mental. } \\
\text { Aprender a trabajar con } \\
\text { otros agentes. Poner } \\
\text { límites con los presos } \\
\text { Aprender a adaptar los } \\
\text { recursos al grupo y a su nivel. } \\
\text { Servicio } \\
\text { Rompe la monotonía en la vida } \\
\text { de los presos, permitiéndoles } \\
\text { socializar. Mejora las funciones } \\
\text { motoras de los presos, así } \\
\text { como el respeto y el auto } \\
\text { control, decreciendo la } \\
\text { competitividad y agresividad en } \\
\text { los juegos. Además de mejorar } \\
\text { los hábitos de higiene. } \\
\text { Programa: Bien valorado, } \\
\text { programado y con objetivos, } \\
\text { permite cambiar dinámicas en } \\
\text { la vida del centro }\end{array}$ \\
\hline
\end{tabular}


Aprendizaje-Servicio universitario en contextos de actividad física, educación física y deporte...

\begin{tabular}{|c|c|c|c|c|c|c|c|c|}
\hline 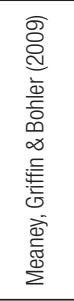 & 蛋 & $\begin{array}{l}\text { Niños de } \\
\text { educación infantil } \\
\text { que no reciben } \\
\text { educación física } \\
\text { por limitaciones } \\
\text { económicas }\end{array}$ & $\begin{array}{l}\text { Semanas: } 8 \\
\text { № de veces a la } \\
\text { semana: Dos veces } \\
\text { a la semana } \\
\text { Horas/sesión: - } \\
\text { Horas totales: - }\end{array}$ & 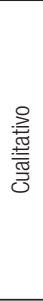 & 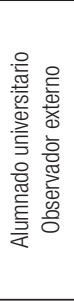 & 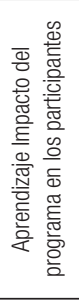 & $\begin{array}{l}\text { Diarios escritos } \\
\text { Entrevistas } \\
\text { semiestructuradas al } \\
\text { alumnado universitario } \\
\text { Observación de la clase } \\
\text { para valorar la actividad } \\
\text { física de los niños }\end{array}$ & $\begin{array}{l}\text { Mejora del conocimiento } \\
\text { pedagógico del alumnado } \\
\text { Emociones positivas y } \\
\text { negativas durante la } \\
\text { intervención } \\
\text { Servicio: niños implicados altos } \\
\text { niveles de AFMV }\end{array}$ \\
\hline 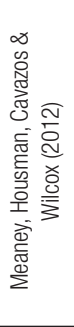 & 胥 & $\begin{array}{l}\text { Niños (5-12 años) } \\
\text { en escuelas de } \\
\text { primaria de zonas } \\
\text { de bajos ingresos }\end{array}$ & $\begin{array}{l}\text { Semanas: } 3 \\
\text { No de veces a la } \\
\text { semana: Una vez a } \\
\text { la semana } \\
\text { Horas/sesión: } 2 \\
\text { horas } \\
\text { Horas totales: - }\end{array}$ & 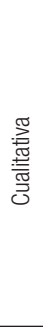 & 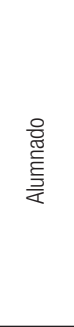 & 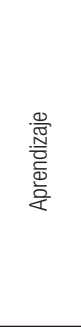 & $\begin{array}{l}\text { Diarios narrativos } \\
\text { reflexivos semanales } \\
\text { Grupos de discusión }\end{array}$ & $\begin{array}{l}\text { Emociones: pensamientos } \\
\text { negativos (ansiedad, } \\
\text { depresión, nerviosismo) } \\
\text { que se disipó con el tiempo } \\
\text { Mejora autoeficacia, confianza } \\
\text { en sí mismo) } \\
\text { Cambio de pensamientos y } \\
\text { actitudes hacia personas en } \\
\text { contextos desfavorecidos }\end{array}$ \\
\hline 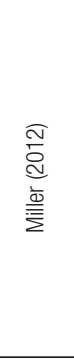 & 姿 & $\begin{array}{l}\text { Intervenciones } \\
\text { con } 33 \text { niños de } \\
3-5 \text { años (12 de } \\
\text { ellos en Educación } \\
\text { Especial) }\end{array}$ & $\begin{array}{l}\text { Semanas: } 8 \\
\text { № de veces a la } \\
\text { semana: una vez a } \\
\text { la semana } \\
\text { Horas/sesión: 45' } \\
\text { Horas totales: - }\end{array}$ & $\frac{\sqrt{x}}{2}$ & 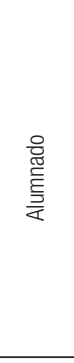 & 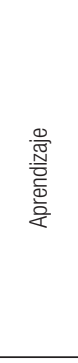 & $\begin{array}{l}\text { Registros semanales } \\
\text { estructurados. } \\
\text { Cuestionario Vocación } \\
\text { para enseñar } \\
\text { del profesorado } \\
\text { de EF (Diseñado } \\
\text { específicamente para } \\
\text { esta investigación). }\end{array}$ & $\begin{array}{l}\text { Reafirmación importancia de la } \\
\text { profesión } \\
\text { Influencia positiva en la } \\
\text { preparación para atender al } \\
\text { alumnado con necesidades } \\
\text { educativas especiales } \\
\text { Estado de desaliento se reduce } \\
\text { a lo largo de intervención; el de } \\
\text { emoción y alegría se mantiene }\end{array}$ \\
\hline 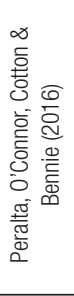 & 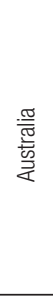 & Niños aborígenes & $\begin{array}{l}\text { Semanas: } 2 \\
\text { № de veces a la } \\
\text { semana: Dos veces } \\
\text { a la semana } \\
\text { Horas/sesión: 120' } \\
\text { Horas totales: - }\end{array}$ & $\frac{\frac{0}{x}}{\sum}$ & 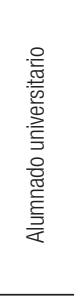 & 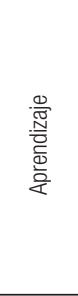 & $\begin{array}{l}\text { Cuestionario Multicultural } \\
\text { Teaching Competencies } \\
\text { Scale Reflexiones } \\
\text { individuales y en grupo. } \\
\text { Grupos de discusión. }\end{array}$ & $\begin{array}{l}\text { Mejora a lo largo del ApS. } \\
\text { Cambio de estereotipos y } \\
\text { nociones preconcebidas } \\
\text { Competencia docente: } \\
\text { dificultad en aplicar una } \\
\text { enseñanza centrada en el } \\
\text { alumnado y las dificultades en } \\
\text { la gestión de la clase }\end{array}$ \\
\hline 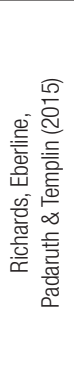 & 蛋 & $\begin{array}{l}\text { Niños con } \\
\text { discapacidad } \\
\text { (3-18 años) }\end{array}$ & $\begin{array}{l}\text { Semanas: } 5 \\
\text { No de veces a la } \\
\text { semana: Una vez a } \\
\text { la semana } \\
\text { Horas/sesión: } 2 \\
\text { horas } \\
\text { Horas totales: - }\end{array}$ & . & 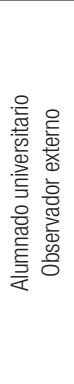 & 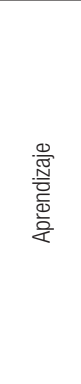 & $\begin{array}{l}\text { Public Affairs } \\
\text { Scale (Escala de } \\
\text { asuntos públicos) } \\
\text { Entrevistas semi- } \\
\text { estructuradas } \\
\text { Diarios reflexivos } \\
\text { semanales } \\
\text { Observaciones no } \\
\text { participantes }\end{array}$ & $\begin{array}{l}\text { Mejora en el compromiso } \\
\text { con la comunidad, liderazgo } \\
\text { ético, competencia } \\
\text { cultural... Consideran } \\
\text { que su participación en } \\
\text { el programa supone un } \\
\text { cambio en la comunidad } \\
\text { Conectar aprendizajes previos } \\
\text { en la práctica } \\
\text { Apertura de nuevas vías } \\
\text { laborales }\end{array}$ \\
\hline 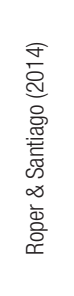 & 胥 & $\begin{array}{l}\text { Estudiantes con } \\
\text { discapacidad }\end{array}$ & $\begin{array}{l}\text { Semanas: - } \\
\text { No de veces a la } \\
\text { semana: - } \\
\text { Horas/sesión: - } \\
\text { Horas totales: } 6 \\
\text { horas. }\end{array}$ & 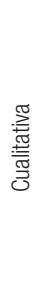 & 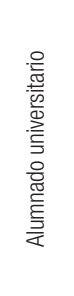 & 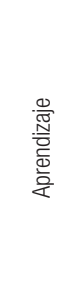 & $\begin{array}{l}\text { Grupo de Discusión } \\
\text { Entrevistas semi } \\
\text { estructuradas }\end{array}$ & $\begin{array}{l}\text { Aparición de nervios, miedo } \\
\text { al principio. Cambio de las } \\
\text { actitudes preconcebidas y } \\
\text { cómo estas cambian con la } \\
\text { experiencia }\end{array}$ \\
\hline
\end{tabular}




\begin{tabular}{|c|c|c|c|c|c|c|c|c|}
\hline 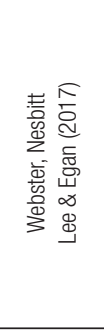 & 胥 & $\begin{array}{l}\text { Estudiantes } \\
\text { de primaria } 0 \\
\text { secundaria }\end{array}$ & $\begin{array}{l}\text { Semanas: } 10 \\
\text { No de veces a la } \\
\text { semana: Dos veces } \\
\text { a la semana } \\
\text { Horas/sesión: } 3 \\
\text { horas } \\
\text { Horas totales: - }\end{array}$ & 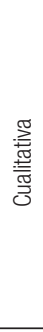 & 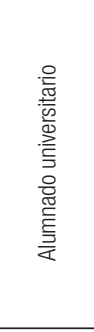 & 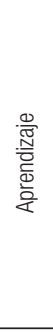 & Entrevistas y reflexiones & $\begin{array}{l}\text { Mejora habilidad comunicativa } \\
\text { Competencia docente: } \\
\text { preparación y planificación }\end{array}$ \\
\hline 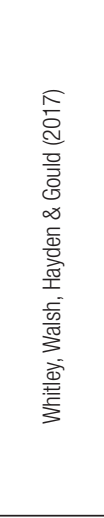 & 胥 & $\begin{array}{l}\text { Estudiantes } \\
\text { de instituto de } \\
\text { comunidades } \\
\text { desatendidas }\end{array}$ & $\begin{array}{l}\text { Semanas: } 12 \\
\text { № de veces a la } \\
\text { semana: dos veces a } \\
\text { la semana } \\
\text { Horas/sesión: - } \\
\text { Horas totales: - }\end{array}$ & 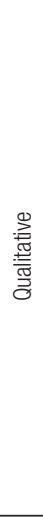 & 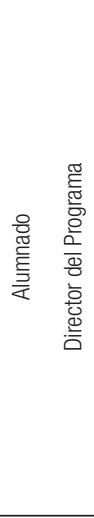 & 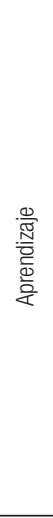 & $\begin{array}{l}\text { Diario reflexivo semanal } \\
\text { Entrevista con el director } \\
\text { del programa } \\
\text { Entrevistas semi- } \\
\text { estructuradas con los } \\
\text { participantes }\end{array}$ & $\begin{array}{l}\text { Mejora autoconfianza, } \\
\text { seguridad en sí mismo, } \\
\text { liderazgo } \\
\text { Reducción de creencias } \\
\text { estereotipadas; Mejora del } \\
\text { conocimiento de la justicia } \\
\text { social y mayor valor al } \\
\text { compromiso cívico } \\
\text { Conocimientos específicos del } \\
\text { contenido, comprensión de los } \\
\text { principios teóricos } \\
\text { Exploración de posibilidades } \\
\text { docentes y laborales }\end{array}$ \\
\hline 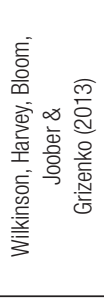 & 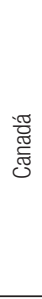 & $\begin{array}{l}\text { Niños con TDAH } \\
\text { (6-12 años) }\end{array}$ & $\begin{array}{l}\text { Semanas: - } \\
\text { № de veces a la } \\
\text { semana: - } \\
\text { Horas/sesión: - } \\
\text { Horas totales: } 16 \\
\text { horas. }\end{array}$ & 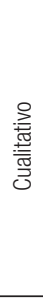 & 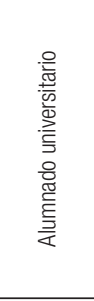 & 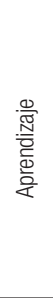 & $\begin{array}{l}\text { Entrevistas semi- } \\
\text { estructuradas }\end{array}$ & $\begin{array}{l}\text { Mejora del Conocimiento } \\
\text { pedagógico del contenido } \\
\text { Desarrollo profesional }\end{array}$ \\
\hline 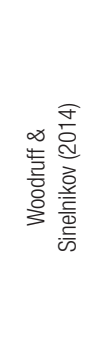 & 胥 & $\begin{array}{l}\text { Adultos con } \\
\text { discapacidad } \\
\text { intelectual }\end{array}$ & $\begin{array}{l}\text { Semanas: } 10 \\
\text { № de veces a la } \\
\text { semana: - } \\
\text { Horas/sesión:1 h 25' } \\
\text { Horas totales: - }\end{array}$ & 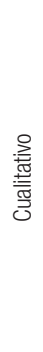 & 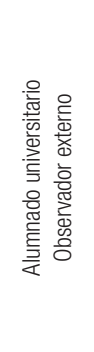 & 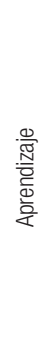 & $\begin{array}{l}\text { Entrevistas, incidentes } \\
\text { críticos, reflexiones } \\
\text { formales y observación } \\
\text { directa }\end{array}$ & $\begin{array}{l}\text { Al principio de miedo, } \\
\text { aprensión, que cambiaron con } \\
\text { el paso del programa. } \\
\text { Cambio en la percepción sobre } \\
\text { la discapacidad cambió. } \\
\text { Competencia docente: } \\
\text { desarrollar propuestas } \\
\text { centradas en el aprendizaje } \\
\text { individualizado }\end{array}$ \\
\hline
\end{tabular}

Fuente: Elaboración propia.

\section{Características de los programas de intervención}

\section{Población receptora del servicio}

Respecto al tipo de receptores del servicio, por un lado, tenemos que, en función de la edad, un 64\% de las investigaciones llevan a cabo el ApS con niños (3-12 años), un 8\% con jóvenes (12-18 años), un $16 \%$ con niños y jóvenes, y un 4\% con familias. Solo un 8\% se desarrollan con adultos y ninguna con adultos mayores (60 o más años). 
Respecto a la diversidad de receptores, encontramos que un 36\% de las intervenciones de ApS se llevan a cabo con participantes con diversidad funcional, por ejemplo, discapacidad, parálisis cerebral, trastornos del lenguaje, motóricos, etc., siendo en estos casos todos niños. Un 8\% de las intervenciones se llevan a cabo con niños con Trastorno por Déficit de Atención e Hiperactividad (TDAH) y un 4\% con adultos con discapacidad intelectual. Un $28 \%$ se llevan a cabo en zonas de bajos ingresos o contextos desfavorecidos. Una de las intervenciones (4\%) se lleva a cabo con reclusos de una unidad psiquiátrica y otra de las intervenciones con niños de educación infantil que por falta de recursos su centro no puede ofertar Educación Física. Por último, un 16\% de las intervenciones no reportan ninguna dificultad o característica especial de la población receptora del servicio.

\section{Duración de los programas de intervención}

Respecto a la duración de las intervenciones de ApS, la información facilitada en cada una de las investigaciones es muy diversa. Cuatro de las investigaciones no reportan información sobre la duración del programa de ApS, el número de intervenciones del alumnado o las horas de servicio realizadas. De los restantes 21 estudios, 8 no presentan datos sobre el número de semanas. Las investigaciones que sí recogen esta información, los programas van desde las 2 a las 15 semanas con un 53.85\% de las intervenciones teniendo una duración de menos de 10 semanas, y un 45.15\% más de 10 semanas. Con relación al total de horas de los proyectos, de los 21 estudios que proporcionan información de la duración, solo 8 presentan esta información. Tres de estos tienen más de 40 horas de duración, y los 5 restantes menos de 20 horas.

Respecto al número de semanas de desarrollo del proyecto de ApS y el número de intervenciones semanales, encontramos 10 investigaciones que no presentan esta información. De las restantes, 10 investigaciones presentan intervenciones de ApS de entre 1-10 semanas y 5 estudios intervenciones de entre 10 y 15 semanas.

\section{Tipo de investigación}

\section{Método de las investigaciones}

De las investigaciones analizadas, 2 de ellas utilizan una metodología cuantitativa, 14 metodología cualitativa y 9 utilizan un método mixto de investigación.

\section{Instrumentos empleados}

En cuanto a los instrumentos que se utilizan en las investigaciones para la recogida de información encontramos que un 52\% de las investigaciones emplean 3 o más instrumentos. En los estudios cuantitativos, los cuestionarios empleados son muy variados buscando responder a diferentes propósitos en cada investigación. En la investigación de carácter cualitativo, destaca la utilización del diario reflexivo o las reflexiones escritas 
$(n=17)$ con preguntas o indicadores de reflexión destinados a recoger información sobre los aspectos que se quieren valorar en la intervención. Destaca también el uso de las entrevistas $(n=15)$ en la mayoría de los casos de carácter semi-estructurado.

\section{Qué información se recoge}

Un 76\% de las investigaciones analizadas centran su foco de atención únicamente en el aprendizaje del alumnado universitario. La información recogida en las mismas es muy diversa dependiendo de los objetivos propuestos en la investigación. Va desde el desarrollo de competencias curriculares (conocimiento pedagógico del contenido), competencias sociales (trabajo en equipo, colaboración con diferentes agentes), competencias culturales y cívicas (atención a la diversidad y a las diferencias individuales, compromiso social) y aprendizajes sobre el desarrollo e identidad profesional. Del resto de investigaciones, un 12\% incluyen resultados del aprendizaje y del servicio, un 8\% del aprendizaje, servicio y del programa implementado, y un 4\% del aprendizaje del alumnado y del programa. Ninguno de los estudios recoge información del servicio o del programa de forma exclusiva.

\section{A quién se recoge la información}

Respecto a los agentes de los que se recoge la información para las investigaciones, destaca la participación del alumnado universitario en el 100\% de las mismas. De estas, 15 recogen en exclusiva la visión del alumnado. Los 10 restantes presentan una gran variedad de combinaciones. Tres de ellos incluyen al alumnado y un observador externo, 2 al alumnado y a los receptores del servicio, otra al alumnado y al responsable de la entidad y otras 2 a todos los agentes participantes (alumnado, receptores, representantes de la entidad y docente universitario), otra al alumnado, los receptores y un observador externo y una última al alumnado, los representantes de la entidad y el docente universitario.

\section{Resultados encontrados}

Por último, en este apartado se recogen los resultados que se derivan de las investigaciones. Estos se han dividido en investigaciones que han valorado los efectos del ApS sobre el aprendizaje del alumnado universitario, y aquellas que se centran en los beneficios obtenidos en el servicio o la evaluación del programa implementado.

\section{Aprendizaje del alumnado universitario}

Para sintetizar los aprendizajes del alumnado universitario estos se han agrupado estos en 6 grandes categorías que aparecen de forma recurrente en la investigación:

(a) Competencia curricular/docente: Dieciséis estudios presentan resultados relativos a este tipo de aprendizaje. La mayoría encuentran que se valora muy positivamente el aprendizaje experiencial, pudiendo poner en práctica los aprendizajes curriculares. 
Investigaciones como las de Galvan et al. (2018), Gil-Gómez et al. (2015), Meaney et al. (2009), Whitley et al. (2017) y Wilkinson et al. (2013), reportan una percepción de mejora del conocimiento pedagógico del contenido. Por otra parte, la investigación también ha mostrado mejoras en la preparación y planificación de las clases (CHIVA-BARTOLL et al., 2018a; WEBSTER et al., 2017) y en la gestión del aula (CORBATÓN-MARTÍNEZ et al., 2015; GALVAN; PARKER, 2011; LLEIXÀ; RÍOS, 2015), así como las dificultades encontradas en la misma por parte del alumnado (PERALTA et al., 2016). También aparecen dificultades a la hora de desarrollar propuestas centradas en el aprendizaje individualizado y centrado en el alumnado (PERALTA et al., 2016; WOODRUFF; SINELNIKOV, 2014), o en ocasiones la falta de conocimiento de contenido para llevar a cabo las propuestas (GALVAN; PARKER, 2011). Por último, la investigación de Lamoneda (2018) encuentra una buena percepción del desarrollo de las competencias docentes.

(b) Competencia social: las investigaciones principalmente han destacado la mejora en aspectos relacionados con la colaboración y comunicación con otros agentes (CHIVABARTOLL et al., 2018a; CHIVA-BARTOLL et al., 2018b; CUEVAS-GOTERRIS et al., 2016; GALVAN et al., 2018; LLEIXÄ; RÍOS 2015; WEBSTER et al., 2017), sin embargo, no aparecen resultados positivos para la mejora del trabajo en equipo (CUEVAS-GOTERRIS et al., 2016). La investigación de Whitley et al. (2011) encuentra mejoras en el liderazgo del alumnado. Entre las investigaciones algunas no encuentran resultados remarcables para la mejora de la competencia social (GALVAN; PARKER, 2011; GIL-GÓMEZ et al., 2015).

(c) Competencia cultural y cívica: la principal información encontrada en relación con esta competencia es el cambio de las actitudes preconcebidas del alumnado universitario hacia la diversidad, la discapacidad y los contextos con dificultades, eliminando las creencias estereotipadas CAPELLA-PERIS et al., 2015; CORBATÓN-MARTÍNEZ et al., 2015; CUEVAS-GOTERRIS et al., 2016; DOMANGUE; CARSON, 2008; GALVAN et al., 2018; GIL-GÓMEZ et al., 2015; GIL-GÓMEZ et al., 2016; LLEIXÀ; RÍOS 2015; MEANEY et al., 2012; PERALTA et al., 2016; ROPER; SANTIAGO, 2014; WHITLEY et al., 2017; WOODRUFF; SINELNIKOV, 2014). Solo una de las investigaciones no muestra diferencias en la competencia cultural tras el ApS (CRANDALL; ZACHARY, 2013).

(d) Desarrollo profesional: las investigaciones revisadas han revelado la importancia del ApS para reafirmar la vocación por la profesión (CAPELLA-PERIS et al., 2015; MILLER, 2012) y para explorar nuevas posibilidades laborales (CAPELLA-PERIS et al., 2014; RICHARDS et al., 2015; WHITLEY et al., 2017)

(e) Identidad personal: la participación en los programas de ApS ha mostrado también mejoras en la autoconfianza y la seguridad en uno mismo (CHIVA-BARTOLL et al., 2018b; CORBATÓN-MARTÍNEZ et al., 2015; HIMELEIN et al., 2010; MEANEY et al., 2012; WHITLEY et al., 2017).

(f) Sentimientos: Por último, 4 de las investigaciones incluidas en la revisión valoran los sentimientos que la participación en los programas de ApS genera en el alumnado, destacando todas que al principio el alumnado se encuentra nervioso, aprensivo o con miedo, pero estas sensaciones se van disipando a medida que avanzan en la intervención (MEANEY et al., 2009; 2012; MILLER, 2012; ROPER; SANTIAGO, 2014; WOODRUFF; SINELNIKOV, 2014). 


\section{Servicio}

Los resultados encontrados en las 4 investigaciones que estudian el impacto del ApS en los receptores del servicio, son variados. Galvan et al. (2018) encuentran mejoras en la resistencia cardiorrespiratoria de los niños con los que intervienen; Meaney et al. (2009) muestran que los niños implicados en las actividades de actividad física se involucran durante las mismas en altos niveles de actividad física moderada-vigorosa; el estudio de Himelein et al. (2010) reporta que se ha percibido un impacto en el estilo de vida de las familias, aumentando la actividad física y mejorando los hábitos alimenticios; por último, el estudio de Lleixà y Ríos (2015) encuentran que el ApS llevado a cabo con reclusos de la unidad de enfermedad mental contribuye a romper la monotonía en la vida de los presos, permitiéndoles socializar con gente de fuera, y además, mejora las funciones motoras de estos, hábitos de higiene, el respeto y el auto control, decreciendo la competitividad y agresividad en los juegos.

\section{Discusión}

La presente revisión recoge los artículos publicados sobre ApS universitario y educación física, actividad física o deporte entre 2008 y 2018 que cumplen los criterios de inclusión propuestos. Esta revisión ha mostrado el incremento en el interés que se ha producido por el ApS en propuestas de actividad física en los últimos años, aunque a través de propuestas de intervención muy variadas en cuanto a población, duración, tipo de investigación, instrumentos para recoger la información y aspectos estudiados. Esto permitirá avanzar y ampliar los horizontes en la investigación en ApS, abordando todos los aspectos que hasta ahora no se han investigado.

Los contextos de intervención han destacado por centrarse en población en edad escolar, apenas valorando el funcionamiento de propuestas de ApS con adultos o adultos mayores. Sin embargo, el alumnado de las titulaciones de actividad física y deporte debe adquirir competencias también en el trabajo con estos grupos poblacionales, donde los intereses, el tipo y la forma de las intervenciones difieren del trabajo con niños y jóvenes. Por otra, existe un amplio número de estudios en los que los receptores del servicio son personas con diversidad funcional o en contextos de pobreza o con pocos recursos, siendo únicamente el estudio de Lleixà y Ríos (2015) el que aborda una intervención con un grupo poblacional de diferentes características, en este caso con presos de una unidad psiquiátrica. Nuevamente, parece que la diversidad de las intervenciones es insuficiente teniendo en cuenta las diferentes vías laborales a las que el alumnado universitario puede acceder, no abordándose contextos con mujeres en riesgo de exclusión social, adultos mayores, personas sin hogar, poblaciones de diferentes etnias, inmigrantes o en grupos poblacionales de riesgo por padecer algún tipo de enfermedad (diabetes, cáncer, etc).

Adicionalmente, el $68 \%$ de las investigaciones no incluye nada de información sobre el número total de horas realizadas en el servicio, y en las que presentan esta información existe una gran diversidad en la duración de las intervenciones. Esto dificulta valorar cómo interviene el factor tiempo en los resultados encontrados. This makes it 
difficult to assess how the time factor influences the results found. Obviously, one would expect to see differences in the levels of achievement in the aims of the proposal between interventions of 60 hours, compared with a practice of only 5 hours.

En la metodología de estas investigaciones predomina sobre todo la triangulación de información a través de la utilización de varios instrumentos (un 52\% de las investigaciones emplean 3 o más instrumentos), pero no a través de la triangulación de la perspectiva de todos los agentes. De las investigaciones analizadas solamente 2 recogen la valoración de todos los agentes implicados en el proyecto de ApS GALVAN; PARKER, 2011; LLEIXÀ; RÍOS, 2015). Únicamente uno de estos dos valora el aprendizaje, el servicio y el programa. Esto parece una limitación de las investigaciones presentadas por las características de la propia metodología, ya que el requisito de cubrir una necesidad social y dar respuesta a sus objetivos hace que el papel que juegan los receptores y las entidades sea fundamental para valorar globalmente la eficacia de la aplicación de esta metodología (BLOUIN; PERRY, 2009). El ApS no puede quedarse únicamente en valorar los efectos que sobre el aprendizaje del alumnado tiene su utilización, sino que igual de importante es valorar qué se estén consiguiendo los objetivos que con el programa se proponen, si se cubre la necesidad social detectada, y validar el propio programa como elemento para dar respuesta a esa necesidad (TRYON; STOECKER, 2008). Para conseguir este objetivo una evaluación objetiva y sistemática de estas necesidades es necesaria.

La revisión sistemática realizada ha mostrado que el ApS puede ser una metodología útil para el aprendizaje y el desarrollo personal del alumnado universitario. El ApS en contextos de actividad física, educación física o deporte se realiza con aprendizajes experienciales pudiendo poner la teoría trabajada previamente en contextos de práctica reales, contribuye a mejorar las habilidades comunicativas del alumnado al tener que coordinar su trabajo con otros agentes, favorece el cambio de percepciones e ideas preconcebidas hacia colectivos vulnerables o en riesgo de exclusión social y contribuye a reafirmar o abrir nuevas vías profesionales. Esta visión coincide con las investigaciones realizadas sobre ApS universitario en otros contextos (HÉBERT; HAUF, 2015).

La fortaleza de esta revisión radica en el amplio número de estudios incluidos en la misma, así como en la profundidad con la que se han abordado los elementos más importantes para poder valorar los resultados obtenidos en las investigaciones de ApS en contextos de actividad física, educación física y deporte. Otra fortaleza es la inclusión de estudios publicados tanto en español como en inglés, permitiendo una visión más amplia del ApS a nivel internacional y mostrando las diferencias entre proyectos implementados en diferentes regiones. Sin embargo, los resultados de esta investigación están limitados por los criterios de inclusión establecidos en la revisión porque no se han incluido propuestas que simplemente describan el desarrollo de proyectos de ApS. Además, es imposible comparar claramente los resultados obtenidos por los diferentes estudios debido a la diversidad de métodos y técnicas utilizadas para la recolección de información, así como a las características particulares de los programas de ApS, impidiendo sacar conclusiones contundentes sobre sus resultados.

Futuras líneas de investigaciones deben ahondar en aspectos que no han sido abordados en profundidad hasta el momento, como: (1) la especificidad de los conocimientos 
del área, por ejemplo, competencias concretas sobre el conocimiento del contenido sobre todo; (2) valorar más las competencias de intervención en contextos; (3) los cambios en las competencias cívicas y sociales, no solo a corto sino a largo plazo; (4) las competencias específicas de intervención con colectivos con características especiales, como personas con discapacidad y la capacidad para proponer tareas adaptadas a sus necesidades; y (5) los efectos concretos que los programas tienen sobre los colectivos en función de los objetivos que se proponían, como la mejora de los niveles AF, hábitos saludables, ocupación activa del tiempo libre, mejora autoestima, socialización, etc. Además de plantear una línea de investigación más fuerte "controlando" los diferentes aspectos de la intervención (duración, colectivos) y utilizando instrumentos con mayor consistencia.

\section{Conclusión}

El ApS universitario en contextos de actividad física, educación física y deporte ha mostrado los numerosos beneficios que aporta a los estudiantes a nivel académico, social y laboral; y que puede aportar a los receptores contribuyendo a que puedan aprovecharse de los beneficios de la práctica de actividad física, mejorando su salud y motricidad. Como evidencia este estudio, el potencial de la metodología SL como propuesta formativa sugiere ampliar su uso en el contexto universitario, ampliar la variedad de proyectos desarrollados en esta área y desarrollar el servicio con otros colectivos con propuestas de actividad física más diversas. Las investigaciones futuras también deben ampliarse para abordar la perspectiva de todos los agentes involucrados en los proyectos e incluir una explicación más rigurosa de las características de los proyectos que permitan dilucidar el impacto real de los resultados obtenidos.

\section{Referencias}

BLOUIN, David; PERRY, Evelyn. Whom does Service-Learning really serve? Community-based organizations' perspectives on Service-Learning. Teaching Sociology, v. 37, n. 2, p. 120-135, 2009. D0I: https://doi. org/10.1177/0092055X0903700201

BRINGLE, Robert; CLAYTON, Patti. Civic education through service learning: What, how, and why? In: MCILRATH, Lorraine; LYONS, Ann; MUNCK, Ronaldo (ed.). Higher education and civic engagement: comparative perspectives. New York: Palgrave Macmillan, 2012. p. 101-124.

CAPELLA-PERIS, Carlos; GIL-GÓMEZ, Jesús; MARTÍ-PUIG, Manuel. La metodología del aprendizaje-servicio en la educación física. Apunts. Educación Física y Deportes, Cataluña, v. 116, n. 2, p. 33-43, 2014. D0I: http://dx.doi.org/10.5672/apunts.2014-0983.es.(2014/2).116.03

CAPELLA-PERIS, Carlos et al. Multiple-case study with life histories in the early childhood education degree: service-learning in the teaching of physical education. Profesorado, Granada, v. 19, n. 1, p. 334-348, 2015.

CAPELLA-PERIS, Carlos; GIL-GÓMEZ, Jesús; MARTÍ-PUIG, Manuel. La metodología del aprendizaje-servicio en la educación física. Apunts. Educación Física y Deportes, Cataluña, v. 116, n. 2, p. 33-43, 2014. D0I: http://dx.doi.org/10.5672/apunts.2014-0983.es.(2014/2).116.03 
CAPELLA-PERIS, Carlos et al. Multiple-case study with life histories in the early childhood education degree: service-learning in the teaching of physical education. Profesorado, Granada, v. 19, n. 1, p. 334-348, 2015.

CHIVA-BARTOLL, Óscar; CAPELLA-PERIS, Carlos, PALLARĖS-PIQUER, Marc. Investigación-acción sobre un programa de aprendizaje-servicio en la didáctica de la educación física. Revista de Investigación Educativa, Murcia, v. 36, n. 1, p. 277-293, 2018a. D0l: http://dx.doi.org/10.6018/rie.36.1.270581

CHIVA-BARTOLL, Óscar; PALLARÉS-PIQUER, Marc; GIL-GÓMEZ, Jesús. Service-learning and effective personality in physical education preservice teachers. Revista Complutense de Educación, Madrid, v. 29, n. 1, p. 181-197, 2018b. D0l: https://doi.org/10.6018/rie.37.2.303331

CORBATÓN-MARTíNEZ, Raquel et al. Efectos académicos, culturales, participativos y de identidad del ApS en futuros maestros a través de la Educación Física. Profesorado, Granada, v. 19, n. 1, p. 280-297, 2015.

CRANDALL, Jason, ZACHARY, Christopher. The efficacy of service-learning in health education to increase pre-service teachers' openness to diversity. Kahperd Journal, Kentucky, v. 51, n. 1, p. 8-16, 2013.

CUEVAS-GOTERRIS, Elena; CHIVA-BARTOLL, Óscar; FRANCISCO-AMAT, Andrea. Análisis sobre la personalidad eficaz en alumnado de didáctica de la educación física a través de Aprendizaje-Servicio. Revista Inclusiones, Santiago de Chile, v. 3, n. 2, p. 152-169, 2016.

DOMANGUE, Elizabeth; CARSON, Russel. Preparing culturally competent teachers: service-learning and physical education teacher education. Journal of Teaching in Physical Education, v. 27, 347-367, 2008. DOl: https://doi.org/10.1123/jtpe.27.3.347

FURCO, Andrew, NORVELL, Katrina. What is service-learning? Making sense of the pedagogy and practice. In: ARAMBURUZZBBALA, Pilar; MCILRATH, Lorraine; OPAZO, Héctor (coord.). Embedding service-learning in European higher education: developing a culture of civic engagement. New York: Routledge, 2019. p. 13-36.

GALVAN, Christine, MEANEY, Karen; GRAY, Virginia. Examining the reciprocal nature of service-learning for underserved students and preservice teachers. Journal of Teaching in Physical Education, v. 37, p. 363372, 2018. D0I: https://doi.org/10.1123/jtpe.2018-0051

GALVAN, Christine; PARKER, Melissa. Investigating the reciprocal nature of service-learning in physical education teacher education. Journal of Experiential Education, v. 34, n. 1, p. 55-70, 2011. D0l: https:// doi.org/10.1177/105382591103400105

GARDNER, Janet; EMORY, Jan. Changing students' perceptions of the homeless: A community servicelearning experience. Nurse Education in Practice, United Kingdom, v. 29, p. 133-136. D0I: https://doi. org/10.1016/j.nepr.2018.01.001

GIL-GÓMEZ, Jesús; CHIVA-BARTOLL, Óscar; MARTÍ-PUIG, Manuel. The impact of service learning on the training of pre-service teachers: Analysis from a physical education subject. European Physical Education Review, United Kingdom, v. 21, n. 4, p. 467-484, 2015. D0l: https://doi.org/10.1177/1356336X15582358 
GIL-GÓMEZ, Jesús et al. Una experiencia de aprendizaje-servicio en futuros docentes: desarrollo de la competencia social y ciudadana. Revista Complutense de Educación, Madrid, v. 27, n. 1, p. 53-73, 2016. DOI: https://doi.org/10.5209/rev_RCED.2016.v27.n1.45071

HARRINGTON, Keneisha. Addressing the impacts of service-learning on communities: a recommendation report. Georgia: University of Georgia., 2014.

HÉBERT, Ali; HAUF, Petra. Student learning trough service-learning: effects on academic development, civic responsibility, interpersonal skills and practical skills. Active Learning in Higher Education, v. 16, n. 1, p. 37-49, 2015. DOl: https://doi.org/10.1177/1469787415573357

HIMELEIN, Melissa; PASSMAN, Liz; PHILLIPS, Jessica. College teaching and community outreaching: service learning in an obesity prevention program. American Journal of Health Education, v. 41, n. 6, p. 368-378, 2010. DOl: https://doi.org/10.1080/19325037.2010.10599166

LAMONEDA, Javier. Programas de ApS en estudiantes de ciclo formativo en animación y actividad física. Journal of Sport and Health Research, Andalucía, v. 10, n. 1, p. 65-78, 2018.

LOUGH, Benjamin. Global service-learning in institutions of higher education: concerns from a community practice. Globalisation, Societies and Education, United Kingdom, v. 16, n. 1, p. 66-77, 2018. D0I: https://doi.org/10.1080/14767724.2017.1356705

LLEIXÀ, Teresa; RÍOS, Mercedes. Service-learning in physical education teacher training. physical education in the modelo prison, Barcelona. Qualitative Research in Education, v. 4, n. 2, p. 106-133, 2015. D0I: http://dx.doi.org/10.17583/qre.2015.1138

MEANEY, Karen; GRIFFIN, Kent; BOHLER, Heidi. Service-learning: a Venue for enhancing pre-service educators' knowledge base for teaching. International Journal for the Scholarship of Teaching and Learning, Georgia, v. 3, n. 2, p. 1-17, 2009. D0l: https://doi.org/10.20429/ijsotl.2009.030221

MEANEY, Karen S. et al. Examining service-learning in a graduate physical education teacher education course. Journal of the Scholarship of Teaching and Learning, Indiana, v. 12, n. 3, p. 108-124, 2012. DOl: https://doi.org/10.20429/ijsotl.2009.030221

MILLER, Marybeth. The role of service-learning to promote early childhood physical education while examining its influence upon the vocational call to teach. Physical Education \& Sport Pedagogy, United Kingdom, v. 17, n. 1, p. 61-77, 2012. DOl: 10.1080/17408981003712810.

PERALTA, Louisa R. et al. Pre-service physical education teachers' indigenous knowledge, cultural competency and pedagogy: a service-learning intervention. Teaching Education, United Kingdom, v. 27, n. 3, p. 248-266, 2016. DOl: https://doi.org/10.1080/17408981003712810

RICHARDS, K. Andrew et al. Experiential learning through a physical activity program for children with disabilities. Journal of Teaching in Physical Education, v. 34, p. 165-188, 2015. DOl: https://doi. org/10.1123/jtpe.2014-0015 
ROPER, Emily; SANTIAGO, José A. Influence of service-learning on kinesiology students' attitudes toward P-12 Students With Disabilities. Adapted Physical Activity Quarterly, v. 31, p. 162-180, 2014. D0l: https://doi.org/10.1123/apaq.2013-0086

SIEDENTOP, Daryl. Content knowledge for physical education. Journal of Teaching in Physical Education, v. 21, n. 4, p. 368-377, 2002. D0l: https://doi.org/10.1123/jtpe.21.4.368

TRYON, Elizabeth; STOECKER, Randy. The unheard voices: community organizations and service-learning. Journal of Higher Education Outreach and Engagement, v. 12, n. 3, p. 47-60, 2008.

WALKER, Sharryn Larsen. Influencing professional practice through academic service learning. Literacy Practice and Research, v. 45, n. 1, p. 1-26, 2020.

WEBSTER, Collin et al. Preservice physical education teachers' service-learning experiences related to comprehensive school physical activity programming. Journal of Teaching in Physical Education, v. 36, p. 430-444, 2017. DOl: https://doi.org/10.1123/jtpe.2016-0191

WHITLEY, Meredith A. et al. Narratives of experiential learning: students' engagement in a physical activitybased service-learning course. Journal of Teaching in Physical Education, v. 36, n. 4, p. 419-429, 2017. D0l: https://doi.org/10.1123/jtpe.2016-0141

WILKINSON, Shawn et al. Student teacher experiences in a service-learning project for children with attention-deficit hyperactivity disorder. Physical Education and Sport Pedagogy, United Kingdom, v. 18, n. 5, p. 475-491, 2013. D0l: https://doi.org/10.1080/17408989.2012.690385

WOODRUFF, Elizabeth; SINELNIKOV, Oleg. Teaching young adults with disabilities through service learning. European Physical Education Review, United Kingdom, v. 21, n. 3, p. 292-308, 2014. D0I: https://doi. org/10.1177/1356336X14564171

Recebido em: 01.05. 2020

Aprovado em: 01.09.2020

Laura Cañadas es profesora en el Departamento de Educación Física, Deporte y Motricidad Humana. Doctorado con Mención Internacional en Ciencias de la Actividad Física y el Deporte. Sus líneas de investigación se centran en la evaluación formativa, la formación de profesorado y la educación física. 